\title{
In vitro evaluation of the inhalable quercetin loaded nanoemulsion for pulmonary delivery
}

\begin{abstract}
ABSTRCT
Bioavailability of quercetin, a flavonoid potentially known to combat cancer, is challenging due to hydrophobic nature. Oil-in-water $(\mathrm{O} / \mathrm{W})$ nanoemulsion system could be used as nanocarrier for quercertin to be delivered to lung via pulmonary delivery. The novelty of this nanoformulation was introduced by using palm oil ester/ricinoleic acid as oil phase which formed spherical shape nanoemulsion as measured by transmission electron microscopy and Zetasizer analyses. High energy emulsification method and D-optimal mixture design were used to optimize the composition towards the volume median diameter. The droplet size, polydispersity index, and zeta potential of the optimized formulation were $131.4 \mathrm{~nm}, 0.257$, and $51.1 \mathrm{mV}$, respectively. The formulation exhibited high drug entrapment efficiency and good stability against phase separation and storage at temperature $4{ }^{\circ} \mathrm{C}$ for 3 months. It was discovered that the system had an acceptable median mass aerodynamic diameter $(3.09 \pm 0.05$ $\mu \mathrm{m})$ and geometric standard deviation $(1.77 \pm 0.03)$ with high fine particle fraction $(90.52 \pm 0.10 \%)$, percent dispersed $(83.12 \pm 1.29 \%)$, and percent inhaled $(81.26 \pm 1.28 \%)$ for deposition in deep lung. The in vitro release study demonstrated that the sustained release pattern of quercetin from naneomulsion formulation up to $48 \mathrm{~h}$ of about $26.75 \%$ release and it was in adherence to Korsmeyer's Peppas mechanism. The cytotoxicity study demonstrated that the optimized nanoemulsion can potentially induce cyctotoxicity towards A549 lung cancer cells without affecting the normal cells. These results of the study suggest that nanoemulsion is a potential carrier system for pulmonary delivery of molecules with low water solubility like quercetin.
\end{abstract}

Keyword: Palm oil ester; Nanoemulsion; Quercetin; Pulmonary delivery; D-optimal mixture design. 